\title{
STICHERUS LANUGINOSUS (GLEICHENIACEAE, POLYPODIOPSIDA) EN ARGENTINA Y PARAGUAY
}

\author{
Saúl Páez (D), Héctor A. Keller (iD) \& Esteban I. Meza-Torres
}

Instituto de Botánica del Nordeste, Consejo Nacional de Investigaciones Cientificas y Técnicas, Universidad Nacional del Nordeste, Casilla de Correo 209, 3400, Corrientes, Argentina; paezsaul@conicet.gov.ar (autor corresponsal).

\begin{abstract}
Páez, S.; H. A. Keller \& E. I. Meza-Torres. 2021. Sticherus lanuginosus (Gleicheniaceae, Polypodiopsida) in Argentina and Paraguay. Darwiniana, nueva serie 9(2): 387-392.

This work provides data on the distribution of Sticherus lanuginosus in Argentina and Paraguay. A description, illustrations, ecological observations and the first record of this species in Argentina are also provided. With this, the number of species of the genus in Argentina rises to three.
\end{abstract}

Keywords. Distribution; ferns; Flora of Argentina; Flora of Paraguay; Misiones Province; subtropical forest; Sticherus.

Resumen. Páez, S.; H. A. Keller \& E. I. Meza-Torres. 2021. Sticherus lanuginosus (Gleicheniaceae, Polypodiopsida) en Argentina y Paraguay. Darwiniana, nueva serie 9(2): 387-392.

En este trabajo se suministra datos sobre la distribución de Sticherus lanuginosus en Argentina y Paraguay. Se brinda además una descripción, ilustraciones, observaciones ecológicas y el primer registro de la especie en Argentina. Con esto, el número de especies del género en Argentina asciende a tres.

Palabras clave. Bosque suptropical; distribución; Flora de Argentina; Flora de Paraguay; helechos; Provincia de Misiones; Sticherus.

\section{INTRODUCCIÓN}

Gleicheniaceae C. Presl es una familia de helechos reconocibles por sus frondes divididas pseudodicotómicamente y por la presencia de una yema en la base de la lámina, la cual puede quedar latente o desarrollarse (Andersen \& Øllgaard, 1996). Esta familia comprende siete géneros con aproximadamente 160 especies, principalmente tropicales o subtropicales (Liu et al., 2020). En Argentina están registrados los géneros Dicranopteris Bernh. y Sticherus C. Presl (Yañez \& Arana, 2016), ambos de distribución pantropical y con cerca de 20 y 95 especies respectivamente (Lima \& Salino, 2018; PPG I, 2016).

El género Sticherus se caracteriza principalmente por presentar yemas escamosas en sus frondes, sin pinnas menores en la base de la lámina, pinnadopinnatífidas, venas generalmente 1-furcadas y esporas monoletes. En el Neotrópico se presentan 54 especies y tres híbridos (Gonzales \& Kessler, 2011). En Argentina este género estaba registrado con dos especies, Sticherus cryptocarpus (Hook.) Ching y $S$. quadripartitus (Poir.) Ching, ambas presentes en los bosques andino-patagónicos (Yañez \& Arana, 2016). El género también está registrado en países limítrofes con Argentina, en las áreas cercanas o fronterizas. De este modo, está presente en Chile (Aysén del General Carlos Ibáñez del Campo, Biobío, La Araucanía, Los Lagos, Los Ríos, Magallanes y Antártica Chilena, Maule, Nuble), en Bolivia (Cochabamba, La Paz, Santa Cruz), en Brasil (Paraná, Rio Grande do Sul, Santa Catarina), en Paraguay (Canindeyú) y en 
Uruguay (Rivera) (Gonzales \& Kessler, 2011; Lima \& Salino, 2018; Lima \& Salino, 2020; Ponce et al., 2008; Rodríguez, 1995).

El género crece en lugares abiertos, perturbados, en bosques montanos y claros en la vegetación, también en terrenos pantanosos con Sphagnum, zonas de deslizamientos de tierra y bordes de caminos; asimismo se encuentran en los páramos húmedos o arbustivos. Son plantas epipétricas o terrestres, creciendo en superficies arenosas o fangosas. En ocasiones se forman comunidades de varias especies del género (Gonzáles \& Kessler, 2011).

En el marco de la revisión de las Gleicheniaceae de Argentina y Paraguay, se revisaron herbarios de ambos países, estudio que dio como resultado parcial extensiones de área para este género. El objetivo de esta contribución es brindar nuevos datos sobre la distribución de Sticherus lanuginosus (Fée) Nakai en Paraguay y documentar por primera vez su presencia en Argentina.

\section{MATERIALES Y MÉTODOS}

Se revisaron todos los especímenes de la familia Gleicheniaceae de los herbarios BA, BAB, CTES, FCQ, LP, PY, SI (Thiers, 2021). Además, se analizaron imágenes de alta resolución disponibles en los repositorios de Flora del Conosur (http:// www.darwin.edu.ar), GBIF (https://www.gbif. org), INCT-Herbário Virtual da Flora e dos Fungos (INCT-HVFF) (https://specieslink.net) y REFLORA (https://reflora.jbrj.gov.br), correspondientes a especímenes de los herbarios B, BM, COR, F, FURB, HUCS, HUEM, MA, MBM, MG, NY, RB, SJRP, UPCB, US, VIC. Para su determinación a nivel específico, fueron confrontados con las imágenes de los especímenes tipo disponibles en el repositorio Global Plants de Jstor (https://plants.jstor. org/). Se analizaron los caracteres de la forma de la lámina, disposición de los segmentos, indumento, y morfología de esporas. La terminología utilizada para realizar las descripciones morfológicas es la propuesta por Andersen \& Øllgaard (1996).

Los detalles morfológicos fueron obtenidos mediante un microscopio óptico Leica CME con cámara incorporada. Las esporas fueron analizadas sin tratamiento químico previo, con un microscopio electrónico de barrido (MEB) marca JEOL 5800 LV.
Las esporas analizadas fueron extraídas del ejemplar Keller \& Franco 4121 (CTES) y caracterizadas según la terminología propuesta por Morbelli (1980).

El mapa de distribución en Argentina y Paraguay se realizó mediante la aplicación SimpleMappr (Shorthouse, 2010).

\section{RESULTADOS}

Sticherus lanuginosus (Fée) Nakai, Bull. Natl. Sci. Mus. Tokyo 29: 20. 1950. Gleichenia lanuginosa Fée, Crypt. Vasc. Brésil 1: 202. 1869. TIPO: BRASIL. Bahía: sin datos, Blanchet 3706 (lectotipo P [P00625760] foto!, designado por Lima \& Salino 2018). Figs. 1 A-J, 2.

Gleichenia pennigera var. lanuginosa T. Moore, Index Fil. 20: 381. 1862, nomen nudum.

Hierbas erectas, epipétricas o terrestres, de 60-75 cm de alt. Rizomas rastreros de 0,3-0,6 mm de diám., castaño a pardo con escamas castañas, de bases truncadas, acuminadas de $1,5 \mathrm{~mm}$, con márgenes dentados a ciliados hacia el ápice. Frondes remotas, erectas. Pecíolos de 25-35 cm, castaño claro, pajizo, surcado adaxialmente, con escamas similares a las del rizoma, dispersos uniformemente; yema en la base de la lámina, que pueden mantenerse latentes o proliferar a un nuevo raquis, de $0,1-0,3 \mathrm{~cm}$, con escamas castañas, de $2 \mathrm{~mm}$, de ápice filiforme y bordes ciliados. Láminas ramificadas, divididas pseudodicotómicamente 3-5 veces, pinnas de 35$40 \times 20-30 \mathrm{~cm}$, últimas ramificaciones de $7-15 \times$ 0,5-1 cm, pinnatipartidas, cartáceas; raquis y eje central de las últimas ramificaciones surcado adaxialmente, con escamas castañas rojizas de $1,5 \mathrm{~mm}$, de base truncada y bordes ciliados. Segmentos oblongos, de base truncada y ápice levemente acuminado, bordes ligeramente reflexos abaxialmente, venación 1-furcadas, con indumento aracnoideo adaxial en toda la superficie, y abaxial en la vena central, de $0,5 \mathrm{~mm}$ de diám. Soros regularmente circulares, con 3-6 esporangios, ubicados sobre la venación secundaria de la pínnula, distribuidos en una serie a ambos márgenes de la vena central de la pínnula. Esporas monoletes, de superficie psilada, con el diámetro polar de $23,7 \mu \mathrm{m}$ aprox. y el diámetro ecuatorial mayor de $30 \mu \mathrm{m}$ aprox. 


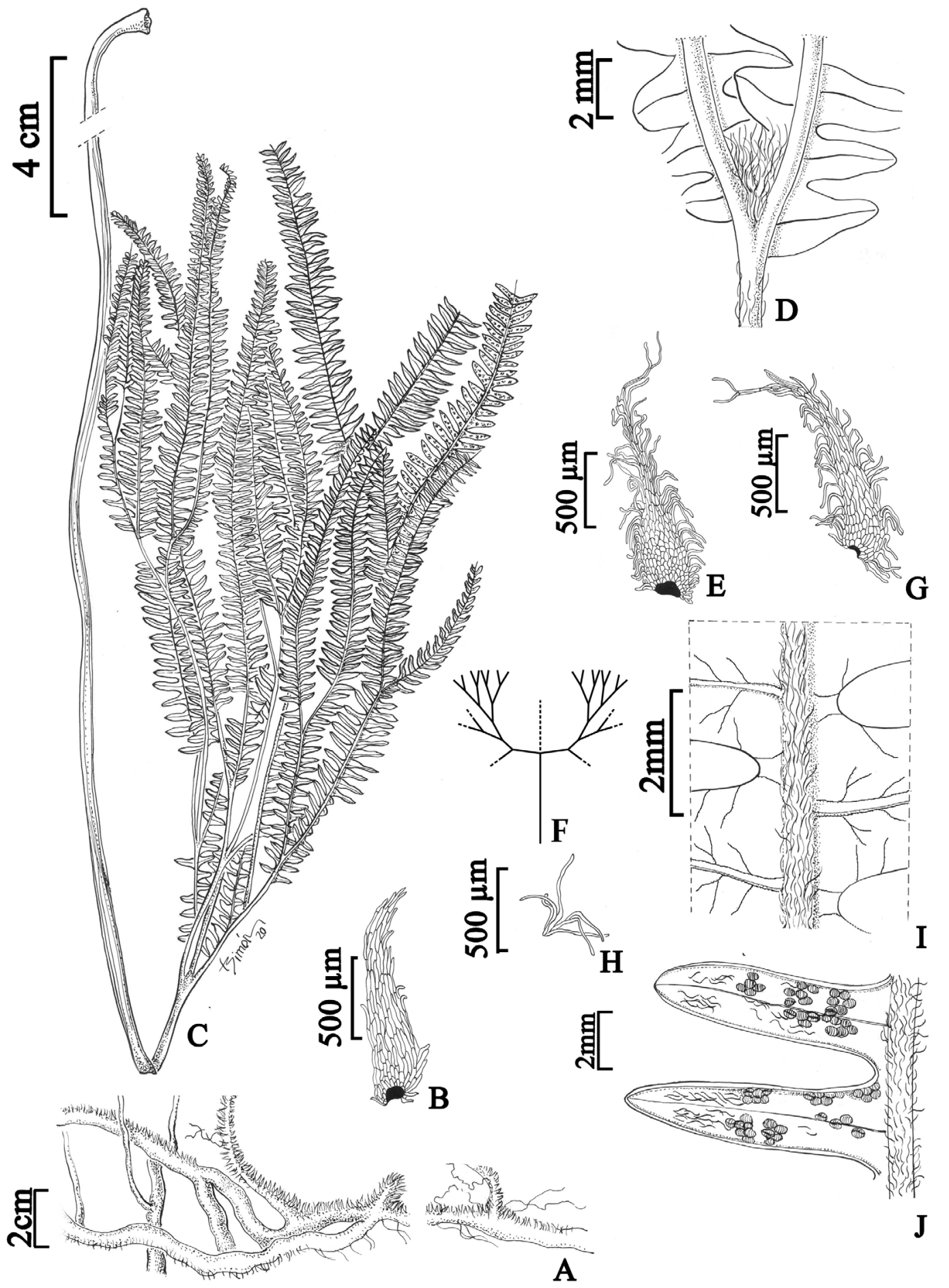

Fig. 1. Sticherus lanuginosus. A, rizoma. B, escama del rizoma. C, fronde completa. D, yema en la base de la lámina, cubierta de escamas. E, escama de la yema de la base de la lámina, en la pseudodicotomía. F, diagrama de ramificación de la lámina. G, escama del eje central de un segmento. H, plano lateral de un indumento aracnoideo del haz del eje central de un segmento. I, detalle de la venación en el hipofilo. J, hipofilo de segmento fértil exhibiendo soros. Keller \& Franco 4121 (CTES). 


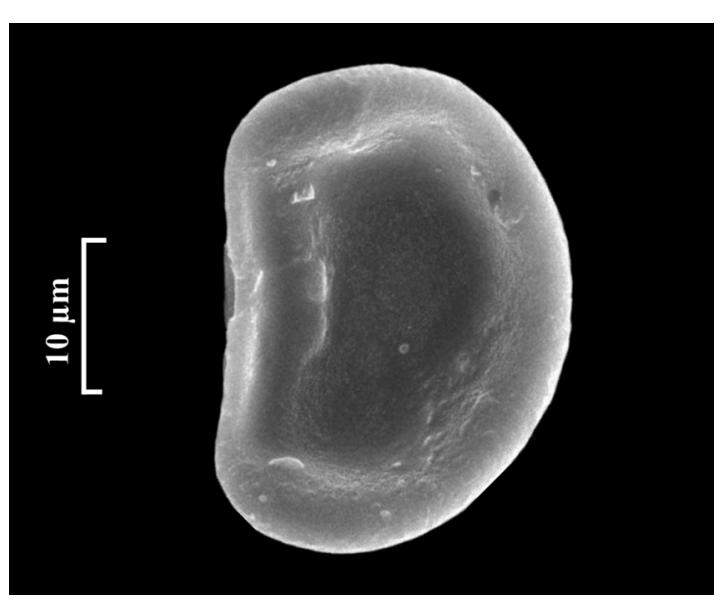

Fig. 2. Detalle de una espora monolete, en vista ecuatorial, de Sticherus lanuginosus (Keller \& Franco 4121, CTES).

Distribución geográfica y hábitat. Centro de Bolivia, Brasil (centro-oeste, sureste, sur del noreste y sur), Colombia, Costa Rica, Ecuador, La Española, Región Oriental de Paraguay, Perú y Venezuela (Lima \& Salino 2020; Ponce et al., 2008; Gonzales \& Kessler, 2011). Habita en pasturas montañosas y sabanas, especialmente en hábitats quemados, 8003000 m s.m. (Kessler \& Smith, 2018). En Argentina se documentó en dos localidades, ambas en la Provincia de Misiones, cercana al límite con Brasil, dentro del Depto. Gral. Manuel Belgrano (Fig. 3). En Paraguay se ha registrado en los departamentos: Alto Paraná [Fiebrig 5753 (foto BM)], Amambay [Hassler 10116 (foto BM)], Caaguazú [Schinini 5828 (CTES)], Canindeyú [Hassler 5147 (foto BM)], Cordillera [Hasller 6811 (foto BM)], Paraguarí [Hahn 2765 (PY)]. Las poblaciones argentinas fueron halladas en claros de bosques secundarios, con vegetación mixta, en ambientes con alteraciones antropogénicas, específicamente en barrancos al borde de rutas y caminos (Fig. 4), en la zona de mayor altitud de la provincia de Misiones (alrededor de $1000 \mathrm{~m} \mathrm{s.m.).}$

Observaciones. En el marco de esta revisión, en Paraguay se ha registrado la presencia en nuevos departamentos, lo que supone una considerable extensión de área de distribución en el centro y este de la Región Oriental de este país, marcada generalmente por sistemas de vegetación característicos del Bosque Atlántico. La revisión de la distribución de la especie en áreas limítrofes en Brasil brinda soporte al hallazgo realizado en este trabajo. Para esto se tomó de referencia a Lima \& Salino $(2018,2020)$.

Sticherus lanuginosus comparte distribución geográfica en Argentina con Dicranopteris flexuosa (Schrad.) Underw., la primera se diferencia por presentar rizoma y base del pecíolo escamoso, indumento en la lámina compuesto por escamas y pelos aracnoideos, y también un porte más robusto. El indumento del rizoma y base del pecíolo está constituido por pelos oscuros y pelos glandulares diminutos, sobre las venas secundarias del hipofilo en $D$. flexuosa.

En áreas cercanas de países limítrofes, próximas a este hallazgo, existen otras especies del género. En Paraguay y Uruguay sólo se encuentra $S$. lanuginosus, mientras que en el sur de Brasil se estima la presencia de cinco especies: Sticherus bifidus (Willd.) Ching, Sticherus gracilis (Mart.) Copel., Sticherus nigropaleaceus (J.W. Sturm) J. Prado \& Lellinger, Sticherus paulistanus (Rosenst.) Copel. y Sticherus pruinosus (Mart.) Ching (Lima \& Salino, 2020). Sticherus lanuginosus se diferencia de estas especies principalmente por presentar escamas con bordes ciliados y/o dentados.

En Argentina el género presenta distribución disyunta, encontrándose en el noreste (Bosque Atlántico) y en el sur (Estepa Patagónica (Islas Malvinas), Bosques Subpolares Magallánicos

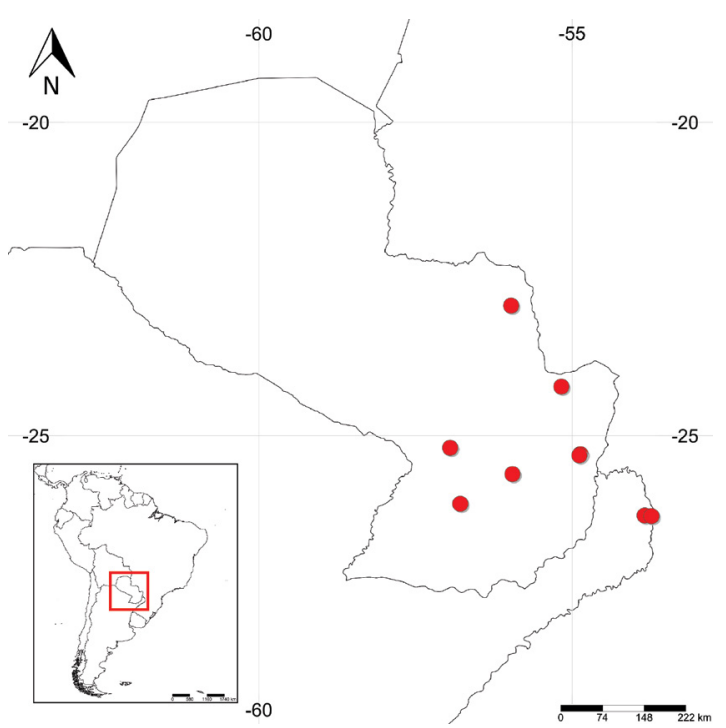

Fig. 3. Mapa de distribución de Sticherus lanuginosus en la Argentina y Paraguay. 


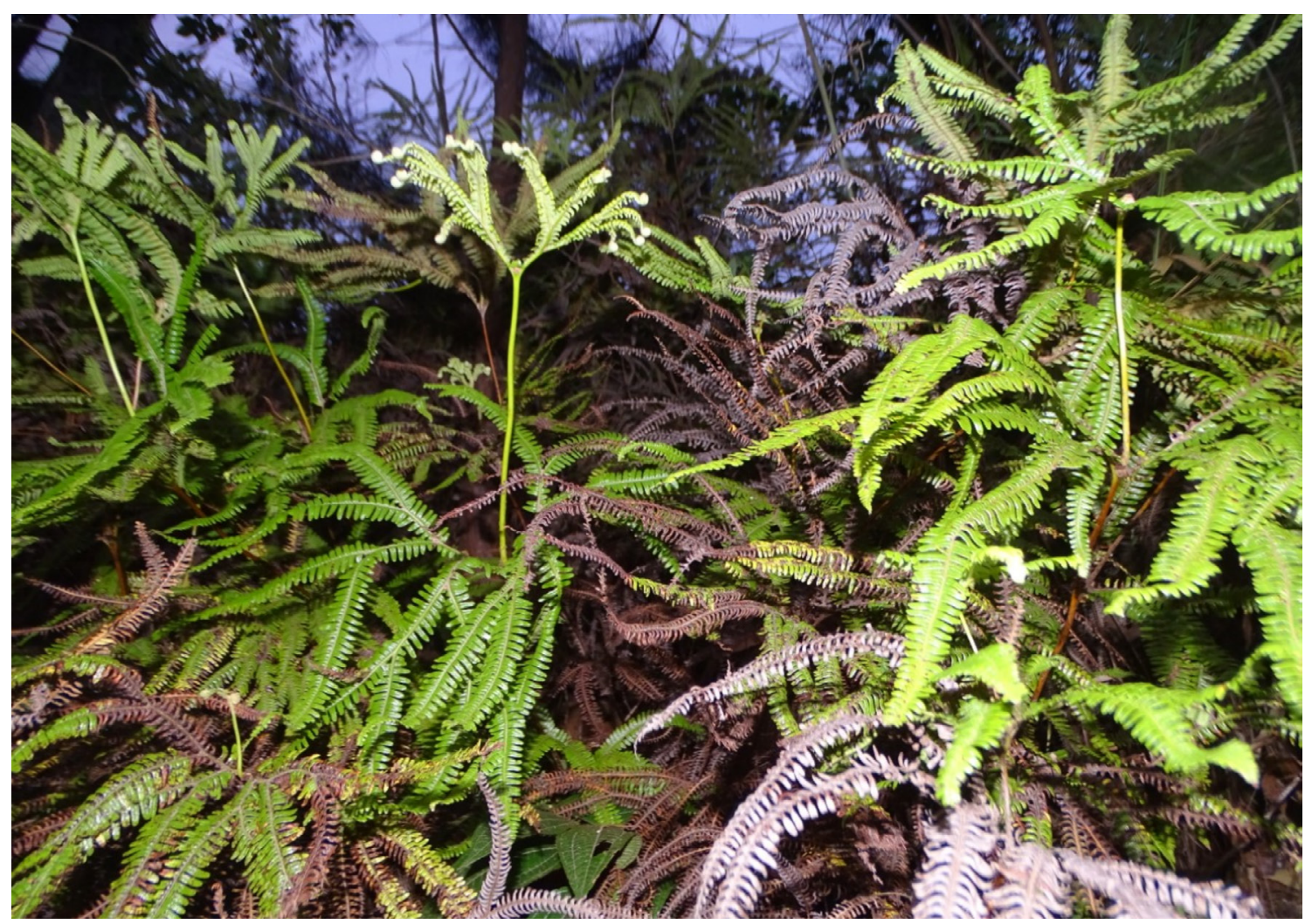

Fig. 4. Hábito de Sticherus lanuginosus, formando una densa población en un barranco en la localidad de Campiñas de Américo, en el Depto. Gral. Manuel Belgrano de la Provincia de Misiones, Argentina. Espécimen de herbario: Keller \& Franco 4121 (CTES). Fotografía: H. A. Keller. Figura en color en la versión en línea http://www.ojs.darwin. edu.ar/index.php/darwiniana/article/view/947/1236

y Bosques Templados Valdivianos). En el sur se encuentran dos especies: Sticherus cryptocarpus (Hook.) Ching y Sticherus quadripartitus (Poir.) Ching, ambas compartidas con Chile. Sticherus lanuginosus se diferencia de éstas por presentar el borde de las pínnulas ligeramente reflexo, poseer un porte más marcadamente ascendente, $\mathrm{y}$ presentar como indumento escamas generalmente triangulares y pelos aracnoideos.

\section{Materiales examinados}

ARGENTINA. Misiones. Depto. Gral. Manuel Belgrano, Campiñas de Américo, alrededores de cementerio, barranco en borde de ruta, rizomatosa, forma matorrales densos, 3-IV-2007, Keller \& Franco 4121 (CTES); Ruta Nac. 14, entre Campiñas de Américo y Bernardo de Irigoyen, puesto de Gendarmería, $26^{\circ} 16^{\prime} 35,42^{\prime}$ S,
5340’32,68”' O, 815 m s.m., 02-X-2019, Keller \& Russell 13915 (CTES); Ruta prov. 17, a $10 \mathrm{~km}$ de Bernardo Irigoyen, afloramiento rocoso, rizomas rastreros, ramificados, $26^{\circ} 17^{\prime} 04^{\prime \prime} \mathrm{S}, 5^{\circ} 44^{\prime} 00^{\prime \prime} \mathrm{O}$, 750 m s.m., 26-IX-2004, Múlgura de Romero et al. 3999 (SI). PARAGUAY. Alto Paraná. In regione fluminis, 1909, Fiebrig 5753 (foto BM). Amambay. In altaplanitie et declivibus "Sierra de Amambay", 1907, Hassler 10116 (foto BM). Caaguazú. Estancia Itá Carú, en campos de Yatay, en pendientes rocosas, a orillas del arroyo, 29-XII-1972, Schinini 5828 (CTES). Canindeyú. In regione Yerbalium de Maracayú. Paraguaria euro-austra, 1898, Hassler 5147 (foto BM). Cordillera. In regione Cordillerae Centralis, 1909, Hasller 6811 (foto BM). Paraguarí. Parque Nacional Ybycuí, bosque deciduo de 15 metros de alto y campos abiertos, planta litófita, 260ㅗ' S, 5640’ O, 05-IX-1984, Hahn 2765 (PY). 


\section{AGRADECIMIENTOS}

El primer autor agradece a la Facultad de Ciencias Exactas y Naturales de la Universidad Nacional de Asunción, por financiar parcialmente una pasantía realizada en el Instituto de Botánica del Nordeste, y a Laura Simón, quien contribuyó con la ilustración del espécimen. Este trabajo fue realizado en el marco del Proyecto "Estudios morfoanatómicos, embriológicos y ecofisiológicos en plantas vasculares americanas" (SECyT-UNNE PI N 20-F013). También obtuvo apoyo del Proyecto PICT No:2016-0531 (Res. 285/17).

\section{BIBLIOGRAFÍA}

Andersen E. Ø. \& B. Øllgaard. 1996. A note on some morphological terms of the leaf in the Gleicheniaceae. American Fern Journal 86: 52-57.

Catálogo de las Plantas Vasculares del Conosur. Instituto de Botánica Darwinion. 2020, http://www.darwin.edu.ar [consulta mayo-octubre 2021].

GBIF (Infraestructura Mundial de Información en Biodiversidad). 2020, https://www.gbif.org [consulta septiembre-octubre 2021].

Global Plants. Jstor. 2019, http://plants.jstor.org [consulta agosto 2021]

Gonzales, J. \& M. Kessler. 2011. A Synopsis of the Neotropical species of Sticherus (Gleicheniaceae) with descriptions of nine new species. Phytotaxa 31: 1-54. DOI: https://doi. org/10.5167/uzh-56305

Herbario Virtual Reflora. Programa Reflora. 2020, https:// reflora.jbrj.gov.br [consulta septiembre-octubre 2021].

Instituto Nacional de Ciência e Tecnologia. Herbário Virtual da Flora e dos Fungos (INCT-HVFF). 2020, https:// specieslink.net [consulta septiembre-octubre 2021].

Kessler, M. \& A.R. Smith. 2018. Prodomus of a Fern flora for Bolivia. XI. Gleicheniaceae. Phytotaxa 344: 53-63. DOI: https://doi.org/10.11646/phytotaxa.344.1.7

Lima, L. V. \& A. Salino. 2018. The fern family Gleicheniaceae (Polypodiopsida) in Brazil. Phytotaxa 358: 199-234. DOI: https://doi.org/10.11646/phytotaxa.358.3.1

Lima, L. V. \& A. Salino. 2020. Gleicheniaceae en Flora do Brasil. Jardim Botânico do Rio de Janeiro. http:// floradobrasil.jbrj.gov.br/reflora/floradobrasil/FB115637 [consulta agosto-octubre 2021.
Liu, H.; F. Rakotondrainibe, S. Hennequin \& H. Schneider. 2020. The significance of Rouxopteris (Gleicheniaceae, Polypodiopsida): a new genus endemic to the Madagascan region. Plant Systematics and Evolution 306: 30 1-11. DOI: https://doi.org/10.1007/s00606-020-01657-9

Morbelli, M. A. 1980. Morfología de las esporas de Pteridophyta presentes en la región Fuegopatagónica, República Argentina. Opera Lilloana 28: 1-138.

Olson, D. M.; E. Dinerstein, E. D. Wikramanayake, N. D. Burgess, G. V. N. Powell, E. C. Underwood, J. A. D'amico, I. Itoua, H. E. Strand, J. C. Morrison, C. J. Loucks, T. F. Allnutt, T. H. Ricketts, Y. Kura, J. F. Lamoreux, W. W. Wettengel, P. Hedao \& K. R. Kassem. 2001. Terrestrial Ecoregions of the World: A New Map of Life on Earth: A new global map of terrestrial ecoregions provides an innovative tool for conserving biodiversity. BioScience 51: 11 933-938. DOI: https://doi. org/10.1641/0006-3568(2001)051[0933:TEOTWA]2.0.CO;2

Ponce, M. M.; R. Rodríguez Ríos \& J. Gonzales. 2008. Gleicheniaceae, en Zuloaga, F. O.; O. Morrone \& M. J. Belgrano (eds.). Catálogo de las plantas vasculares del Cono Sur (Argentina, sur de Brasil, Chile, Paraguay y Uruguay). Monographs in Systematic Botany from the Missouri Botanical Garden 107: 51-55.

PPG I. 2016. A community-derived classification for extant lycophytes and ferns. Journal of Systematics and Evolution 54: 563-603. DOI: https://doi.org/10.1111/jse.12229

Rodríguez, R. R. 1995. Pteridophyta, en Marticorena, C. \& R. R. Rodríguez (eds.), Flora de Chile, Volumen I: 119-337. Ediciones de la Universidad de Concepción, Concepción, Chile.

Shorthouse, D. P. 2010. SimpleMappr, an online tool to produce publication-quality point maps [https://www.simplemappr.net].

Thiers, B. [permanentemente actualizado, consulta 2021]. Index Herbariorum: a global directory of public herbaria and associated staff. New York Botanical Garden's Virtual Herbarium, http://sweetgum.nybg.org/ih

Yañez, A. \& M. D. Arana. 2016. Familia Gleicheniaceae C. Presl, en Ponce, M. M. (autora); A. M. Anton, F. O. Zuloaga \& M. J. Belgrano (eds.), Flora vascular de la República Argentina 2: Licofitas, Helechos, Gymnospermae. Buenos Aires: IBODA-IMBIV, CONICET. pp. 171-174. DOI: https://doi.org/10.2307/j.ctt1p0vjr5

Zanotti, C. A.; H. A. Keller \& F. O. Zuloaga. 2020. Biodiversidad de la flora vascular de la provincia de Misiones, Región Paranaense, Argentina. Darwiniana, nueva serie 8(1): 42291. DOI: https://doi.org/10.14522/darwiniana.2020.81.878 